\section{USO DE LOS BLOGS POLÍTICOS: ANÁLISIS DE ALGUNOS FACTORES DETERMINANTES}

\author{
Stefano De Marco \\ José Manuel Robles Morales \\ Departamento de Sociología III, Fac. CCEE \\ Universidad Complutense de Madrid \\ stefanodemarco@estumail.ucm.es \\ jmrobles@ccee.ucm.es
}

\section{THE USE OF POLITICAL BLOGS: AN ANALYSIS OF SOME KEY FACTORS}

\begin{abstract}
This paper aims to explore the factors that lead an Internet user to type comments on a political blog in more detail. Specifically, we sought to find out what political variables influence this behaviour and what uses of the Internet seem to favour it. We also studied the effect of a number of socio-demographic control variables. To do this, we used the data from the 2007 survey on "The Internet and political participation" (ref. 2736), run by the Centro de Investigaciones Sociológicas. We began with an exploratory factor analysis, which was followed by a logistic regression. In order to exclude influences on the model due to the first-level of digital divide, the sample chosen for the analysis comprised Spanish Internet Users.
\end{abstract}

KEY WORDS: Determinants; political blogs; factor analysis; internet uses; digital divide.

\section{INTRODUCCIÓN}

Hoy en día la academia está dedicando un interés creciente a los efectos de la introducción en el ámbito político de las nuevas tecnologías de la información y comunicación - TIC (Colombo, 2007).

En este ámbito de estudio es posible destacar dos grandes ramas. La primera tiene que ver con los planteamientos teóricos acerca de los posibles desarrollos democráticos que podrian impulsar las TIC (Hacker y Van Dijk, 2001; Subirats, 2002).

Otra corriente de estudio es de tipo empírico y vierte sobre la participación política online sus conexiones con la participación tradicional y las posibles desigualdades que puedan surgir de la no difusión de los usos políticos
RESUMEN: Objetivo de este trabajo es avanzar en la comprensión de los determinantes que hacen que un internauta escriba comentarios en un blog político. Más en concreto, se ha decidido averiguar qué variables políticas influyen sobre este comportamiento y qué usos de Internet parecen favorecerlo. También se ha estudiado el efecto de algunas variables socio-demográficas de control. Para ello, se han utilizado los datos del estudio de 2007 "Internet y participación política" (ref. 2736) del Centro de Investigaciones Sociológicas. Se ha implementado un análisis factorial exploratorio

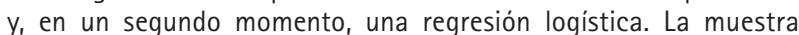
elegida para el análisis ha coincido con los internautas españoles, para así descartar influencias en el modelo debidas a la brecha digital de primer nivel.

PALABRAS CLAVE: Determinantes; blogs políticos; análisis factorial; usos de internet; brecha digital.

de Internet a toda la población. Robles (2007) define la participación política online o activismo digital como el conjunto de las actividades políticas que los ciudadanos realizan a través de Internet.

En este contexto investigativo han primado estudios enmarcados en la teoría de la normalización (Margolis y Resnick, 2000) y los estudios acerca de la brecha digital (DiMaggio, Hargittai, Neuman y Robinson, 2001). Esta última coincide con las diferencias entre la población en términos de recursos informativos entre los que tienen y los que no tienen acceso a Internet. Recientes estudios han demostrado que estas diferencias pueden derivar también de distintos usos de internet entre la población (brecha digital de segundo nivel) o por el hecho de tener o no motivaciones políticas para el uso de Internet (brecha de tercer nivel). 
Por el otro lado, según la teoría de la normalización, las relaciones socioeconómicas y de poder que caracterizan el mundo real se estarian reproduciendo también en el ciberespacio. Esto implicaria que Internet no representaría una oportunidad para la innovación democrática. Muchos trabajos empíricos han sido realizados para confirmar (Bimber, 2001) o negar esta teoria (Schuefele y Nisbet, 2002; Krueger, 2002), principalmente intentando averiguar qué efectos tiene el activismo digital sobre la participación tradicional.

Sin embargo, algunos estudiosos han empezado a plantear la independencia de la participación online, intentando así emancipar este concepto de la participación tradicional. Según esta perspectiva, el activismo digital sería un objeto de estudio per se, que cuenta con mecanismos y recursos peculiares y especificos (Gibson, Lusoli y Ward, 2005; Best y Kreueger, 2005). En relación al uso de blogs políticos, algunos autores empiezan a considerarlo como una nueva forma de participación política. Citando a Anduiza et al.: "¿Puede no considerarse participación el hecho de escribir comentarios políticos en blogs cuando éstos comienzan a tener un papel propio en la definición de la agenda mediática y, por lo tanto, política?" (2010, p. 135).

En este trabajo se pretende avanzar en la comprensión de los determinantes que hacen que un internauta escriba comentarios en un blog político. Siguiendo una perspectiva de investigación meramente exploratoria, se ha decidido averiguar qué variables políticas influyen sobre este comportamiento y qué usos de Internet parecen favorecerlo. También se ha estudiado el efecto de algunas variables socio-demográficas de control.

Para ello, se han utilizado los datos del estudio de 2007 "Internet y participación política" (ref. 2736) del Centro de Investigaciones Sociológicas. La muestra elegida ha coincidido con los internautas españoles, para así descartar influencias en el modelo debidas a la brecha digital de primer nivel.

Se han recogido todas las variables que en este estudio miden las actividades y actitudes políticas estudiadas en la literatura que se ha ocupado de blogs. También se han elegido las variables que miden el consumo de información por parte del sujeto entrevistado.
En primer lugar, para poder ver si la información proporcionada por estas variables era resumible en un número reducido de dimensiones, se ha procedido a un análisis factorial. Los factores extraídos, junto con las variables que miden los distintos usos de Internet y algunas variables socio-demográficas, han sido seleccionados como variables independientes de un modelo de regresión logística. Se ha elegido como variable dependiente una pregunta del estudio 2736 que mide el hecho de escribir comentarios en blogs o páginas web de contenido político.

Este trabajo se desarrollará de la siguiente manera. Primero se esbozará el estado de la cuestión acerca de la investigación sobre Internet y participación política para luego profundizar los trabajos académicos que se han centrado en los blogs. Luego se describirá la naturaleza de los datos y la metodología empleada. Sucesivamente, se presentarán los resultados de los análisis y, finalmente, se comentarán estos resultados intentando enlazarlos con el marco teórico general.

\section{MARCO teóRICO}

El estudio de los efectos en las prácticas políticas y democráticas debidos a la difusión de las nuevas tecnologías de la información y comunicación han sido analizados a partir de dos enfoques principales, uno teórico y el otro empírico.

Según la perspectiva teórica, los autores han planteado posibles escenarios de evolución democrática, generando así diferentes modelos de democracia digital (Subirats, 2002; Hacker y Van Dijk, 2001). Con democracia digital, se entiende el uso de Internet y de las TIC para las funciones de gobierno de una estructura democrática (Dahlberg, 2001). Los diferentes modelos van de ámbitos concretos de actuación, como la e-administración o el e-gobierno, ya puestos en marcha en muchas democracias occidentales, hasta ámbitos potenciales de nuevas formas democráticas alcanzables gracias a las nuevas tecnologías, como por ejemplo la democracia directa.

Según la perspectiva empírica, el debate académico se ha centrado, entre otros temas de investigación, alrededor de dos cuestiones principales: las posibles desigualdades políticas generadas por la brecha digital y la normalización. 
La brecha digital se considera como "el gap tecnológico" entre los que "tienen acceso a la información" y los "que no tienen acceso a ella'" (Attewell, 2000; pág. 252). Las primeras investigaciones se centraban en las desigualdades en el acceso, señalando que los grupos tradicionalmente desfavorecidos manifestaban carencias en el uso de Internet (Dunham 1999). Aunque algunas de estas diferencias se han reducido, la edad, la situación socioeconómica y la raza siguen siendo predictores potentes del uso de Internet (DiMaggio et al., 2001; Warschauer, 2003; Mossberger, Tolbert y Stansbury, 2003). Estudios más recientes se han centrado en el segundo y en el tercer nivel de brecha digital, definidos, respectivamente, por las desigualdades de distribución entre la población de los diferentes usos de Internet, y por las diferencias entre los usuarios políticamente comprometidos y los políticamente indiferentes (Hargittai, 2002; Norris, 2001; Cornfield, 2000). En relación al segundo nivel de brecha digital, Bonfadelli (2002) demuestra que las personas con niveles educativos más altos utilizan Internet de forma más activa y, principalmente, para buscar información. Por otro lado, siempre según el autor, las personas con niveles educativos más bajos tienden a privilegiar los usos de entretenimiento. Según Robinson, DiMaggio y Hargittai (2003), los internautas con niveles educativos más altos se distinguen por usos de Internet que proporcionan ventajas laborales, educativas, de salud y de participación política. Esta tipología de usuarios también mantiene más intercambios de correo electrónico con amigos y familiares $y_{1}$ además, utiliza menos Internet con fines recreativos. Los resultados del trabajo de Wassermann y Richmond-Abbott (2005) sugieren que los hombres son más propensos a utilizar los correos electrónicos para la comunicación profesional y comercial, mientras que las mujeres los utilizan más para la comunicación personal y de larga distancia.

Otro campo de estudio está marcado por la teoría de la normalización. Según los autores de esta teoría, los partidos políticos utilizarían sus páginas web exclusivamente para desempeñar tareas que ya se realizan a través de los medios tradicionales (Margolis y Resnik, 2000; Bimber y Davis, 2003). Además, los partidos con mayor visibilidad en el "mundo real", también la tendrian en el ciberespacio (Gibson y Ward, 2002). Finalmente, esta teoría implicaría que Internet sólo representaría una herramienta más a disposición de los ciudadanos políticamente activos, sin permitir involucrar a los que son políticamente indiferentes (Bimber, 2001).
Los autores de la "nueva movilización", que se oponen a esta teoría, plantean que los usos políticos de Internet pueden favorecer la participación política de algunos colectivos tradicionalmente menos activos, como, por ejemplo, los jóvenes (Livingstone y Helsper, 2007). Además, según algunos autores, tanto las páginas web como los blogs de representantes y partidos políticos presentarian aspectos innovadores para el escenario político, fomentando discusiones y debates de carácter inclusivo y deliberativo (Norris, 2003; Coleman, 2004). Todas estas actividades no se podrían realizar sin las nuevas tecnologías de información y comunicación.

A pesar de la disputa académica, cabe destacar que todos estos estudios se centran en los efectos de la participación política online sobre la participación política tradicional. Sin embargo, recientemente ha surgido una nueva línea de investigación cuyo objetivo es descubrir los elementos distintivos del activismo digital frente a la participación política tradicional. Es decir, se han empezado a estudiar el tipo de recursos (Best y Krueger, 2002) y de actividades (Gibson, Lusoli y Ward, 2005) que caracterizan la participación online.

Este trabajo se enmarca en esta nueva línea de investigación, teniendo como objetivo principal estudiar algunos de los determinantes que influyen en una forma concreta de participación política online, es decir, el uso de blogs o foros de discusión de contenido político.

\subsection{El estudio de los blogs políticos}

A pesar de la creciente atención que la comunidad académica está reservando a los blogs, no hay todavía acuerdo sobre una definición común de esta herramienta. De hecho, muchos autores se centran en descripciones estructurales, según las cuales los blogs serían páginas web compuestas por un conjunto de posts presentados en orden cronológico invertido (Drezner and Farrell, 2004; Nardi et al., 2004). Autores como Bar-Ilan (2005), Johnson y Kaye (2004) o Singer (2005) destacan también la importancia de los enlaces a otros blogs o foros como elemento característico de los blogs, aunque compartido con otras herramientas como las páginas web o los chats. Finalmente, Gil de Zuñiga et al. (2009) definen los blogs como "páginas web interactivas y no sincrónicas que alojan posts centrados en tópicos" (p. 4). Los usuarios pueden poner comentarios

ARBOR Vol. 188756 julio-agosto [2012] 689-705 ISSN: 0210-1963 
y contestar tanto a los posts de los autores como a las intervenciones de los demás usuarios, aunque no todos los blogs lo permitan o algunos requieran registro previo (Gil de Zuñiga et al., 2009).

Los blogs de contenido político tienen que ver con tópicos, eventos y temas políticos de un determinado contexto político a nivel de circunscripción electoral, nacional, internacional o de partido político (Ferguson y Griffiths, 2006).

Según Coleman (2005) uno de los principales campos de aplicación política de los blogs sería el de la información. Por un lado porque los usan los periodistas para colgar artículos que los periódicos para los cuales trabajan no

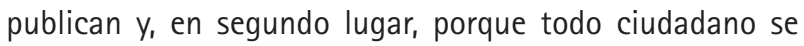
transforma en periodista (Coleman, 2005). Además, los bloggers son vistos como un "quinto poder", desempeñando tareas de control de los medios de comunicación tradicionales (Gillmor, 2004), contrastando los valores preestablecidos de las noticias.

Bruns (2008) plantea el hecho de que los bloggers y los ciudadanos-periodistas reintroducen en el espacio público material que está bloqueado por los canales convencionales. Los medios tradicionales no han conseguido crear una esfera pública unificada que diera lugar a debates sobre temas comunes. Consecuentemente, los blogs permitirian construir distintas esferas públicas sobre distintos temas públicos concretos. En estas esferas publicas múltiples, la gente se informa, habla y busca activamente soluciones políticas (Bruns, 2008). Según Xenos (2008), los blogs políticos también servirian como plataforma para discusiones de carácter deliberativo.

De hecho, los estudios empíricos sobre los efectos del uso de blogs políticos parecen aportar evidencias a favor de una perspectiva que vea estas herramientas más como plataformas participativas que de información. Por ejemplo, según Coleman y Wright (2008), esta tipología de blog puede facilitar la comunicación política de cuatro maneras distintas. En primer lugar, reduciendo la distancia entre el emisor y el lector del mensaje. En segundo lugar, porque facilitan la puesta en marcha de diálogos interactivos, permitiendo al emisor responder directamente al contenido del blog. También permiten a quien escribe mantener una forma de "escucha" que favorece la adecuación del con- tenido de los mensajes al contenido del blog. Finalmente, porque permiten a cada uno de los usuarios proporcionar o comentar noticias (Coleman y Wright, 2008).

Los blogs pueden también ser una herramienta para la formación de opinión, así como de influencia para la agenda política (Drezner y Farrell, 2004). Según Kahn y Kellner (2004), los blogs políticos, organizándose en redes y conectándose entre ellos, están creando una plataforma alternativa a las informaciones vehiculadas a los medios tradicionales. Esta plataforma representaría un instrumento valioso para la movilización de opiniones y para influir sobre las elites (Woodly, 2008).

Según Wallsten (2008), los blogs políticos podrían relacionarse con cuatro funciones. En primer lugar, los blogs políticos se pueden utilizar como "correas de transmisión" que, sin comentarios añadidos del autor, proporcionan enlaces a otras páginas web. En segundo lugar, y en marcado contraste con el concepto de correa de transmisión, los blogs políticos se pueden utilizar como "escenarios" tecnológicos desde los cuales los bloggers pueden transmitir sus opiniones. En tercer lugar, los blogs políticos se pueden utilizar como "movilizadores" que tratan de animar a los lectores a tomar posición política. Por último, los blogs políticos se pueden utilizar para promover nuevos temas de conversación y poder así averiguar las reacciones de los lectores frente a ellos (Wallsten, 2008).

\subsection{Determinantes del uso de los blogs políticos}

Entre los estudios empíricos que se han ocupado de los factores que facilitan el uso de un blog político, cabe destacar el de Johnson y Kaye (2004). Según los autores la credibilidad de los blogs entre los usuarios facilitaría el uso de esta herramienta. Los predictores más importantes de la credibilidad, siempre según los autores, serían las variables políticas, tales como el conocimiento político, el interés hacia la política, la confianza en la política y la participación política.

Johnson et al. (2007) han descubierto que los usuarios de Internet políticamente interesados confían más en los blogs que en cualquier otra fuente de información y que los perciben como más creíbles respecto a las páginas web de los periódicos o que las televisiones online. 
Hwang, Schmierbach, Paek, Gil de Zúñiga y Shah (2006), estudiando los disidentes contra la guerra en Iraq, han encontrado que la disociación del marco propuesto por los medios de comunicación tradicionales constituye una motivación para la búsqueda de información alternativa en Internet, incluso a través de blogs.

Sweetser y Kaid (2008) observaron que los usuarios de blogs políticos pueden tener bajos niveles de cinismo político. Éste se define como un sentimiento general de descontento y desafección hacia las instituciones y los personajes políticos (Miller, 1974; Hershey and Hill, 1975). Siempre en el mismo trabajo, se ha puesto de manifiesto cómo los lectores de blogs no políticos tenían elevados niveles de confianza en la información política y en su capacidad para participar en la política. La eficacia de la información política es un concepto que mide en qué medida confian los ciudadanos en su saber político y en tener los conocimientos necesarios para comprometerse con el proceso político, incluido el voto (Kaid et al., 2007).

También el consumo de noticias puede tener algún efecto sobre el uso de los blogs. Según el estudio de De Zúñiga et al. (2007) esta conducta, junto con la participación en discusiones políticas, fomenta diversas formas de participación. El consumo de noticias, por ejemplo, promueve más conocimiento político, favorece la reflexión y elaboración sobre los medios de comunicación y fomenta un sentido de eficacia política (Eveland, Hayes, Shah, y Kwak, 2005). Estos efectos se han observado en el consumo de noticias a través de periódicos, televisión e Internet, donde la televisión produce efectos más débiles. Escuchar o leer noticias acerca de asuntos públicos también puede proporcionar un recurso para el debate político y crear oportunidades de exposición a puntos de vista no disponibles en la red social (Mutz \& Martin, 2001), fomentando el debate y diálogo que de otra manera no podrían ocurrir.

\section{Metodología}

\subsection{Datos y Muestra}

Los análisis de este trabajo se han hecho a partir de los datos del estudio del CIS "Internet y participación política" (2007, ref. 2736). Esta encuesta es la primera en España que se ha ocupado de recoger información acerca de la difusión de Internet en España y de sus distintos usos, incluidos los políticos, por parte de la población española.

La encuesta cuenta con una muestra de 3.716 sujetos que hacen referencia a un universo constituido por la población española de ambos sexos mayores de 18 años'.

El cuestionario cuenta con un total de 56 preguntas. De éstas, la P.18 y P.19 constituyen filtros para las siguientes preguntas (de P.20 a P.28) sobre los usos de Internet, también los políticos. Para las finalidades de este estudio, se ha decidido descartar de la muestra aquellos sujetos que no sabían lo que es un ordenador personal (P.18) y los que no se habian conectado a Internet en los 3 meses anteriores a la encuesta (P.19). De esta manera, se obtiene una muestra de 2.169 internautas, que es la muestra elegida para nuestro estudio. Esta decisión se motiva por la intención de controlar efectos debidos al primer nivel de brecha digital.

\subsection{Variables independientes}

Las variables introducidas en el análisis factorial se han escogido para poder medir las dimensiones de actitudes políticas (cinismo, eficacia de la información política, interés hacia la política, actitudes ciudadanas y hacia la deliberación), participación política, estímulos movilizadores, sobre todo online (Best y Krueger, 2005), y consumo de información de actualidad y política, tanto online como offline. En la tabla 1 se presentan las variables contenidas en el estudio 2736 y elegidas para este análisis.

La dimensión "Confianza en los blogs" ha sido introducida porque, como se ha mencionado antes, se considera como uno de los factores principales para la utilización de los blogs. A falta de una pregunta explícita en el cuestionario que midiese directamente este constructo, se ha decidido operacionalizarlo a través de tres variables. La primera es la "disposición del voto a través de Internet". Se ha tomado como un índice indirecto de la percepción de la fiabilidad de Internet como plataforma para la participación política (Hsu y Lin, 2008). La "percepción de falta de seguridad de Internet" ha sido introducida con la misma finalidad, pero con puntuación negativa. La variable "Confianza en la gente" se ha tomado como índice indirecto de la posibilidad de confiar

ARBOR Vol. 188756 julio-agosto [2012] 689-705 ISSN: 0210-1963 
TABLA 1. DIMENSIONES E INDICADORES DE ACTITUDES POLITICAS, COMPORTAMIENTOS POLITICOS, CONSUMO DE INFORMACIÓN POLITICA Y ESTIMULOS MOVILIZADORES (ONLINE Y OFFLINE). TIPO DE VARIABLE

\section{DIMENSIÓN}

Consumo de información política

(online y medios tradicionales)

Eficacia de la información política

Interés hacia la política

\section{Cinismo}

Participación política (activismo, participación institucional, participación institucional online)

Estímulos movilizadores

Confianza en Internet/Blog

Actitud hacia deliberación

Actitudes ciudadanas

\section{INDICADORES}

Escucha/ve noticias en radio o televisión

Escucha/ve programas politicos en radio o televisión

Lee prensa internacional

Lee prensa nacional

Lee prensa gratuita

Lee prensa local

Frecuencia uso de Internet para obtener información politica

Estar inscrito a newsletter sobre temas de actualidad politica

Estoy mejor informado sobre política que la mayoría de la gente

La política es complicada de entender

Le resulta difícil decidir para quién ir a votar

Votar es fácil y no requiere ningún esfuerzo

Interés hacia la política

Frecuencia con la que se habla de política

Mi voto es inútil porque no cambia el resultado de las elecciones

Esté quién esté al poder sólo busca sus intereses personales

Ir a una manifestación requiere mucho tiempo y esfuerzo

Ir a una manifestación no sirve para nada

Los políticos no se preocupan de lo que piensa la gente normal

Confianza en el gobierno central

Confianza en los sindicatos

Confianza en los partidos politicos

Confianza en los ayuntamientos

Confianza en los medios de comunicación

Confianza en las ONG

Satisfacción funcionamiento democracia

Uso internet para contactar partido/representante político

Haber consultado página web de un partido/candidato político

Activismo asociativo

Activismo en los últimos 12 meses

Voto en las últimas elecciones

Recibir correos electrónicos de contenido político

Alguien le ha comentado que había una manifestación y le convenció para ir

Ha recibido mensaje de móvil para asistir a manifestación

Disposición voto a través de Internet

Percepción de falta de seguridad de internet

Confianza en la gente

Actitud acerca implicación ciudadana en la toma de decisiones políticas

Importancia cumplir las normas

Importancia no evadir impuestos

Importancia de formarse su propia opinión personal

Importancia votar en las elecciones

Importancia pensar en los demás
TIPO DE

VARIABLE

Ordinal

Ordinal

Ordinal

Ordinal

Ordinal

Ordinal

Ordinal

Dicotómica

Ordinal

Ordinal

Ordinal

Ordina

Ordinal

Ordinal

Ordinal

Ordinal

Ordinal

Ordinal

Ordinal

Numérica

Numérica

Numérica

Numérica

Numérica

Numérica

Numérica

Dicotómica

Dicotómica

Numérica

Numérica

Dicotómica

Numérica

Dicotómica

Dicotómica

Ordinal

Dicotómica

Numérica

Dicotómica

Numérica

Numérica

Numérica

Numérica

Numérica

Fuente: Elaboración propia a partir del cuestionario 2736 del CIS 
en información que no llega de fuentes atendibles, sino de los "periodistas-ciudadanos" (Coleman, 2005). Siendo la perspectiva de este trabajo meramente exploratoria, se ha decidido averiguar si el análisis factorial respalda la construcción de este constructo.

En el análisis de regresión logistica, los factores extraídos anteriormente han sido empleados como variables dependientes.

Con el objetivo de verificar posibles efectos debidos a la brecha digital de segundo nivel, se han introducido en el modelo de regresión logística también las variables que en el estudio 2736 miden los usos de Internet. Estas variables están recogidas en la tabla 2 . Son todas dicotómicas.

TABLA 2. INDICADORES DE LOS USOS DE INTERNET

\begin{tabular}{|l|}
\hline $\begin{array}{l}\text { ¿En los últimos } 12 \text { meses, para cual de las siguientes actividades } \\
\text { ha utilizado Internet? }\end{array}$ \\
\hline Buscar Información (trabajo, estudios, salud, etc.) \\
\hline Comprar algún producto o servicio \\
\hline Realizar gestiones bancarias \\
\hline Recibir o enviar mensajes de correo electrónico \\
\hline Intervenir en chats o foros de discusión \\
\hline Llamar a través de Internet \\
\hline Descargar archivos \\
\hline Mantener su propio blog o página web \\
\hline Navegar por Internet sin un objetivo concreto \\
\hline
\end{tabular}

Fuente: Elaboración propia a partir del cuestionario 2736 del CIS.

Finalmente, se ha decidido introducir en el modelo de regresión algunas variables socio-demográficas de control. Éstas son: sexo, edad, nivel de estudios y estatus socioeconómico.

\subsubsection{Recodificación/cálculo de las variables}

Antes de realizar el análisis, se ha procedido a la recodificación de las variables a introducir.

La pregunta 2.1 ("Escucha/ve noticias en radio o televisión") explica la frecuencia con la que el sujeto ve o escucha las noticias en radio o televisión. Es una variable ordinal. Se han recodificado las puntuaciones asignadas a las categorías de respuestas asignando el valor máximo (4) a la categoría de respuesta "todos los días" y así bajando de puntuación hasta asignar el valor mínimo (0) a la categoria de respuesta "nunca". Siempre de cara al análisis factorial, se han excluido las categorias "no sabe" y "no contesta", a las que se suele atribuir puntuación de 8 y 9, con el riesgo de introducir valores que generen problemas en los resultados del análisis (Pardo, 2005). En la tabla 3 se puede ver cómo estas exclusiones no afectan significativamente al porcentaje válido de sujetos. Todas las demás variables categóricas han sido recodificadas de la misma manera: a la categoría con valor más alto se ha asignado puntuación más alta y así sucesivamente, hasta asignar valor 0 a la categoria menor.

Las variables "Prensa Local", "Prensa Gratuita", "Prensa Nacional" y "Prensa Internacional" son variables ordinales que se han construido a partir de las preguntas $2.3,3.1,3.2,3.3$ y 3.4 del cuestionario ${ }^{2}$. Las tres últimas indican qué tipo de soportes (papel, Internet o ambos) utilizan los sujetos que leen prensa. En primer lugar se ha recodificado la variable 2.3 de la misma manera que la variable 2.1. Sucesivamente se han recodificado las categorias de respuesta de las preguntas $3.1,3.2,3.3$ y 3.4 de tal manera que se asignara puntuación 0 a la categoría de respuesta "No lee". También se ha determinado como valor 1 a las categorias "en papel" e "Internet" y valor 2 a la categoria "ambos". De esta manera se ha querido crear una diferencia cuantitativa entre quien sólo utiliza un soporte y quien lee la prensa en ambos. Finalmente se ha multiplicado la variable 2.3 por cada una de las demás variables. Así, se han perdido sólo los sujetos que han contestado "no sabe" o "no contesta", mientras que se mantienen los que no leen prensa. Las puntuaciones van de 0 (No lee) a 8 (lee en ambos tipo de soporte). Sin embargo no existe un intervalo de tamaño fijo entre cada categoria. De hecho, los valores intermedios coinciden con el $2,3,4$ y 6 .

La variable "Estar inscrito a newsletter sobre temas de actualidad política" es dicotómica, y se ha recodificado asignando valor 0 a la categoría de respuesta "no" y valor 1 a la opción de respuesta "si". Se han excluidos los valores correspondientes a las categorias "no sabe" y "no contesta". Todas las demás variables dicotómicas han sido recodificadas de la misma manera.

ARBOR Vol. 188756 julio-agosto [2012] 689-705 ISSN: 0210-1963

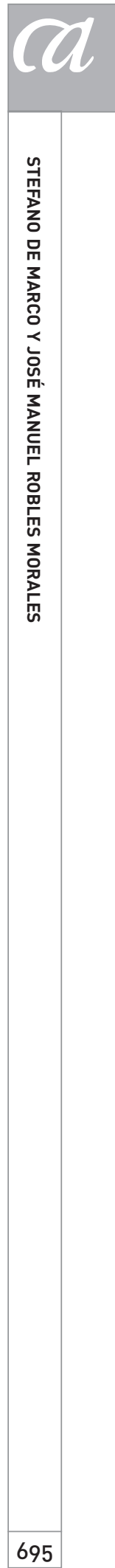

doi: 10.3989/arbor.2012.756n4005 
TABLA 3. CASOS VÁLIDOS Y CASOS PERDIDOS (UNIDADES Y PORCENTAJES SOBRE LA MUESTRA)

\begin{tabular}{|c|c|c|c|c|}
\hline \multirow[t]{2}{*}{ Variables } & \multicolumn{2}{|c|}{ Casos válidos } & \multicolumn{2}{|c|}{ Casos perdidos } \\
\hline & $\mathrm{N}$ & $\%$ & $\mathrm{~N}$ & $\%$ \\
\hline Satisfacción funcionamiento democracia & 2.104 & 97,00 & 65 & 3,00 \\
\hline Confianza en los medios de comunicación & 2.124 & 97,93 & 45 & 2,07 \\
\hline Haber consultado página web de un partido/candidato político & 2.154 & 99,31 & 15 & 0,69 \\
\hline Estar inscrito a newsletter sobre temas de actualidad política & 2.154 & 99,31 & 15 & 0,69 \\
\hline Confianza en la gente & 2.153 & 99,26 & 16 & 0,74 \\
\hline Importancia de formarse su propia opinión personal & 2.098 & 96,73 & 71 & 3,27 \\
\hline Frecuencia uso de Internet para obtener información política & 2.153 & 99,26 & 16 & 0,74 \\
\hline Disposición voto a través de Internet & 2.067 & 95,30 & 102 & 4,70 \\
\hline Actitud acerca implicación ciudadana en la toma de decisiones políticas & 2.090 & 96,36 & 79 & 3,64 \\
\hline Confianza en los partidos políticos & 2.119 & 97,69 & 50 & 2,31 \\
\hline Frecuencia con la que se habla de política & 2.158 & 99,49 & 11 & 0,51 \\
\hline Activismo en los últimos 12 meses & 2.169 & 100,00 & 0 & 0,00 \\
\hline Recibir correos electrónicos de contenido político & 2.169 & 100,00 & 0 & 0,00 \\
\hline Importancia votar en las elecciones & 2.121 & 97,79 & 48 & 2,21 \\
\hline Importancia pensar en los demás & 2.102 & 96,91 & 67 & 3,09 \\
\hline Voto en las últimas elecciones & 2.169 & 100,00 & 0 & 0,00 \\
\hline Percepción de falta de seguridad de Internet & 2.169 & 100,00 & 0 & 0,00 \\
\hline Escucha/ve noticias en radio o televisión & 2.169 & 100,00 & 0 & 0,00 \\
\hline Escucha/ve programas políticos en radio o televisión & 2.161 & 99,63 & 8 & 0,37 \\
\hline Confianza en los sindicatos & 2.040 & 94,05 & 129 & 5,95 \\
\hline Confianza en los ayuntamientos & 2.121 & 97,79 & 48 & 2,21 \\
\hline Confianza en el gobierno central & 2.115 & 97,51 & 54 & 2,49 \\
\hline Importancia no evadir impuestos & 2.109 & 97,23 & 60 & 2,77 \\
\hline Confianza en las ONG & 2.085 & 96,13 & 84 & 3,87 \\
\hline Importancia cumplir las normas & 2.132 & 98,29 & 37 & 1,71 \\
\hline Interés hacia la política & 2.161 & 99,63 & 8 & 0,37 \\
\hline Los políticos no se preocupan de lo que piensa la gente normal & 2.118 & 97,65 & 51 & 2,35 \\
\hline La política es complicada de entender & 2.128 & 98,11 & 41 & 1,89 \\
\hline Esté quién esté al poder sólo busca sus intereses personales & 2.126 & 98,02 & 43 & 1,98 \\
\hline Estoy mejor informado sobre política que la mayoría de la gente & 2.059 & 94,93 & 110 & 5,07 \\
\hline Alguien le ha comentado que había una manifestación y le convenció para ir & 2.138 & 98,57 & 31 & 1,43 \\
\hline Activismo asociativo & 2.169 & 100,00 & 0 & 0,00 \\
\hline Uso internet para contactar partido/representante político & 2.162 & 99,68 & 7 & 0,32 \\
\hline Ha recibido mensaje de móvil para asistir a manifestación & 2.158 & 99,49 & 11 & 0,51 \\
\hline Le resulta difícil decidir para quién ir a votar & 2.099 & 96,77 & 70 & 3,23 \\
\hline Ir a una manifestación no sirve para nada & 2.097 & 96,68 & 72 & 3,32 \\
\hline Votar es fácil y no requiere ningún esfuerzo & 2.122 & 97,83 & 47 & 2,17 \\
\hline Ir a una manifestación requiere mucho tiempo y esfuerzo & 2.097 & 96,68 & 72 & 3,32 \\
\hline Mi voto es inútil porque no cambia el resultado de las elecciones & 2.109 & 97,23 & 60 & 2,77 \\
\hline Lee prensa local & 2.169 & 100,00 & 0 & 0,00 \\
\hline Lee prensa gratuita & 2.169 & 100,00 & 0 & 0,00 \\
\hline Lee prensa nacional & 2.169 & 100,00 & 0 & 0,00 \\
\hline Lee prensa internacional & 2.169 & 100,00 & 0 & 0,00 \\
\hline
\end{tabular}

Fuente: Elaboración propia a partir de los datos del estudio 2736 del CIS. 
La variable "Percepción de falta de seguridad de Internet" se ha construido a partir de la pregunta P31 del cuestionario ("¿Qué inconveniente vería en un sistema de voto a través de Internet?"). Originalmente, ésta era una variable nominal. Se ha decidido asignar puntuación 1 a la categoría de respuesta "La seguridad de estos sistemas no estaría garantizada" y puntuación 0 a las demás categorias de respuestas. La variable resultante de esta recodificación es una variable dicotómica.

Las variables "Activismo en los últimos 12 meses", "Activismo asociativo" y "recibir correos electrónicos de contenido político" son variables numéricas construidas a partir de la suma del conjunto de variables dicotómicas que componen las preguntas P11, P13 y P27, respectivamente. Para ello, se han sumado las respuestas "si" (puntuación 1) a cada ítem de las variables seleccionadas. Las variables resultantes de esta codificación tienen puntuación que va de 0 a " $n$ ", donde " $n$ " es el número de ítems de cada variable. No indican una cantidad, sino el número de distintas conductas puestas en práctica por el sujeto, o el número de distintos tipos de correos políticos recibidos.

La variable "Edad" es numérica. Se ha recodificado eliminando del análisis aquellos sujetos que no han contestado a la pregunta.

Las demás variables numéricas, como, por ejemplo, "Confianza en la gente", son escalas que tienen valor mínimo 0 y valor máximo 10. También en estos casos se ha decidido eliminar las categorías de respuesta "No sabe" y "No contesta".

Las variables "Nivel de estudios" y "Estatus socio-económico" han sido calculadas según los criterios del Centro de Investigaciones Sociológicas. En el primer caso las categorias son: "Sin estudios", "Primaria", "Secundaria", "Formación profesional", "Medios universitarios" y "Superiores". En el segundo caso, las categorias que componen la variable son "Clase alta/media-alta", "Nuevas clases medias", "Viejas clases medias", "Obreros cualificados" y "Obreros no cualificados".

Después de la recodificación se ha analizado el número de casos perdidos por cada variable (Tabla 2).

Así es posible destacar que el porcentaje mínimo de casos válidos es el 94,05 de la variable "Confianza en los sin- dicatos". Es éste un porcentaje de casos válidos suficiente para excluir los casos perdidos "según lista" en el análisis factorial.

\subsection{Variable dependiente}

Para el modelo de regresión logística, se ha decidido utilizar como variable dependiente la pregunta P28(5): " ¿En alguna ocasión ha escrito comentarios en algún foro, blog o pagina web, sobre temas de actualidad, sociales o políticos?". La variable es dicotómica.

Es importante destacar que la pregunta P28(5) no se dirige únicamente a explorar la conducta de escribir comentarios en un blog de contenido político, sino que incluye también "foros y páginas web". Sin embargo, a pesar de la plataforma utilizada, el tipo de conducta se puede considerar equivalente. Cambia el contexto en el que se realiza esta conducta. Consecuentemente el modelo implementado puede que pierda en poder explicativo, debido a la no introducción de factores explicativos inherentes a las demás plataformas.

\subsection{Análisis}

En esta investigación se han utilizado dos herramientas estadisticas distintas. En primer lugar, se ha implementado un análisis factorial que incluyese todas las variables incluidas en el estudio 2736 descritas anteriormente. Esto nos ha permitido, por un lado, resumir la información proporcionada por estas variables $y$, además, explorar las dimensiones según las cuales se organizan.

Debido a la naturaleza de los fenómenos objeto de estudio, algunas de las preguntas incluidas han sido construidas para averiguar bien la presencia o ausencia de una conducta determinada, bien la forma con la que se presenta un determinado fenómeno. Esto implica el uso o de variables dicotómicas o de variables categóricas para la medición de muchas características de los sujetos. De hecho, aplicando la prueba de Kolmogorov Smirnov, se averiguó que todas las variables tenían una distribución significativamente distinta de la distribución normal. Por lo tanto, a la hora de aplicar un análisis factorial (Lewis-Beck, 1994) se descartaron aquellos métodos de extracción de los factores que requieren la multinormalidad de los datos, como lo de máxima verosimilitud. Consecuentemente, se ha optado por el método de extracción de componentes principales

ARBOR Vol. 188756 julio-agosto [2012] 689-705 ISSN: 0210-1963 
(Lawley y Maxwell, 1971). El método de rotación elegido es el varimax.

En un segundo momento, se ha procedido a un análisis de regresión logística que incluía, como variables independientes, los factores extraídos por el análisis anterior, las variables del estudio 2736 que se centran en los distintos usos de internet y algunas variables socio-demográficas de control.

\section{Resultados}

\subsection{Análisis factorial}

En un primer acercamiento exploratorio, se ha permitido al software decidir el numero de factores a extraer, escogiendo aquellos con autovalores mayores o iguales a 1.

Los datos presentados en la tabla 4 nos informan de que la medida de adecuación muestral de Keyser-Meyer-Olkin es mayor de 80 y la prueba de esfericidad de Bartlett es significativa. Además, el determinante de la matriz de correlaciones es suficientemente bajo $(4,27 \mathrm{E} 0,005)$ como para poder indicar intercorrelación entre las variables introducidas en el análisis. Estos índices informan de que es lícito llevar a cabo el análisis factorial.

TABLA 4. PRUEBAS DE ADECUACIÓN DEL ANÁLISIS FACTORIAL A LA MATRIZ DE CORRELACIONES

\begin{tabular}{|l|l|r|}
\hline Índice de Kaiser-Meyer-Olkin &, 847 \\
\hline \multirow{3}{*}{$\begin{array}{l}\text { Test de esfericidad } \\
\text { de Bartlett }\end{array}$} & Chi cuadrado & $1.4767,892$ \\
\cline { 2 - 3 } & Grados de libertad & 903 \\
\cline { 2 - 3 } & Sig. &, 000 \\
\hline
\end{tabular}

Fuente: Elaboración propia a partir del análisis sobre los datos del estudio 2736 del CIS.

Según el criterio de selección de los factores comúnmente conocido como "regla de Keiser" (autovalores $\geq 1$ ) la solución final debería incluir 12 factores, llegando a explicar el $55,49 \%$ de la varianza. La literatura advierte de que este criterio de selección tiende a sobreestimar el número de factores extraídos (Cliff, 1988). Se decidió por lo tanto aplicar un criterio de selección más parsimonioso, el Parallel Test. Este método permitió reducir el número de factores extraídos a 9, que explican globalmente el 48,08\% de la varianza de las variables introducidas.

A falta de un índice de ajuste del modelo, el análisis de los residuos nos informa del hecho de que más del 10\% tiene un $\alpha>0,05$, superando el umbral crítico indicado por Harman (1976). Es razonable pensar que esto sea debido a la naturaleza no numérica de todas las variables introducidas en el modelo.

En definitiva, los resultados del análisis factorial muestran que es posible agrupar las 43 variables escogidas en 9 dimensiones subyacentes que permiten explicar la estructura de covarianza de las variables mismas.

En la página siguiente se presenta la tabla 5 con los componentes rotados que ha servido para la interpretación de los factores.

En primer lugar, cabe destacar que la única dimensión que ha mantenido sus variables después del análisis factorial ha sido la de las Actitudes ciudadanas, que coincide con el factor 5. El factor 1 está compuesto por las variables de la dimensión del cinismo que miden la confianza hacia el gobierno, hacia los partidos y sindicatos, y hacia los medios de comunicación. También está presente satisfacción con el funcionamiento de la democracia. Consecuentemente, este factor se ha denominado Confianza en las instituciones.

El factor 2 incluye variables que miden el interés hacia la política, el consumo de información política a través de la televisión y la frecuencia del uso de Internet para la información política. Se ha interpretado como una Actitud proactiva hacia la política que, sin embargo, no llega a ser una forma de activismo o participación.

El Factor 3 incluye las variables de lectura de periódicos, tanto online como offline, sea prensa nacional, gratuita, local o internacional. Se ha interpretado consecuentemente como Lectura de prensa.

En el cuarto factor contribuyen variables inherentes a la participación institucional, excluyendo el voto, tanto online como offline. También están presentes estímulos de movilización online inherentes a la recepción de correos electrónicos de contenido político. Este factor se ha interpretado como Participación política institucional. 
TABLA 5. FACTORES Y VARIABLES CON COMPONENTES ROTADOS

\begin{tabular}{|c|c|c|c|c|c|c|c|c|c|}
\hline \multirow{2}{*}{ VARIABLES } & \multicolumn{9}{|c|}{ FACTORES } \\
\hline & 1 & 2 & 3 & 4 & 5 & 6 & 7 & 8 & 9 \\
\hline Confianza en el gobierno central & ,769 & ,076 &,- 041 & ,038 & ,090 &,- 125 &,- 007 & ,025 &,- 051 \\
\hline Confianza en los sindicatos & ,765 & 038 & ,001 & ,043 &,- 053 &,- 086 & 090 & ,009 &, 035 \\
\hline Confianza en los partidos políticos & ,737 & .174 &,- 014 &, 122 & ,090 &,- 181 &,- 106 & 074 &,- 047 \\
\hline Confianza en los ayuntamientos & ,728 & 024 & .018 & .037 & 041 &,- 036 &,- 008 & ,035 &, 002 \\
\hline Confianza en los medios de comunicación & ,655 &,- 065 & ,000 &,- 091 & ,026 & ,085 &, 016 & ,003 &,- 009 \\
\hline Confianza en las ONG & .584 &,- 046 & ,003 & ,038 & 089 & ,007 &, 192 &,- 049 &, 017 \\
\hline Satisfacción funcionamiento democracia &, 577 &, 047 & ,009 &,- 062 & 163 &,- 149 &,- 011 &, 099 &,- 166 \\
\hline Frecuencia con la que se habla de política & .035 & ,700 &, 035 & 150 & 104 &,- 191 & 234 &, 110 & ,038 \\
\hline Escucha/ve programas políticos en radio o televisión & ,089 & ,699 &,- 026 & 061 &, 000 & , 106 &,- 023 & 104 &,- 045 \\
\hline Interés hacia la política &, 134 & ,694 &, 031 & 202 & 104 &,- 249 & , 196 & ,079 & ,017 \\
\hline Estoy mejor informado sobre política que la mayoría de la gente &,- 057 &, 548 &,- 030 & 239 & 029 &,- 108 & 026 &,- 047 & ,060 \\
\hline Frecuencia uso de Internet para obtener información política &,- 034 & 483 &, 026 & 440 & 093 &,- 034 & 087 &,- 003 &,- 099 \\
\hline Escucha/ve noticias en radio o televisión & .124 & 449 &,- 014 &,- 137 &,- 022 & 150 &,- 076 & 272 &, 111 \\
\hline Lee prensa internacional & ,009 & ,033 & 835 & ,018 &, 019 & ,038 &,- 011 &,- 006 & 008 \\
\hline Lee prensa nacional &,- 026 & .029 & ,784 &,- 044 & 046 & ,025 & 057 & .016 &,- 073 \\
\hline Lee prensa gratuita &,- 012 &,- 036 & ,781 & .002 & ,033 &,- 057 & ,019 &,- 016 & ,001 \\
\hline Lee prensa local &, 014 &,- 022 &, 696 & .030 &,- 005 &,- 010 & ,009 &,- 012 & ,073 \\
\hline Uso internet para contactar partido/representante político &,- 004 & 068 &,- 012 & 709 &, 004 &,- 040 & 038 & .042 & .047 \\
\hline Estar inscrito a newsletter sobre temas de actualidad política & 024 & .022 &, 015 & ,663 &,- 014 & 011 &,- 001 & 077 & ,040 \\
\hline Haber consultado página web de un partido/candidato político & ,047 & ,308 &,- 006 &, 581 & 051 &,- 088 &, 073 & ,069 &,- 100 \\
\hline Recibir correos electrónicos de contenido político &,- 016 & .166 &,- 030 & ,548 & 011 &,- 096 & ,408 &,- 016 &,- 052 \\
\hline Activismo asociativo &, 086 & , 103 & ,006 & ,363 & ,024 &,- 054 & 239 & , 143 &, 045 \\
\hline Importancia cumplir las normas &, 144 &,- 002 & ,053 &,- 023 & 763 & ,076 &,- 154 & 122 &,- 020 \\
\hline Importancia no evadir impuestos &, 098 & ,052 &, 029 & .029 & 751 &,- 010 &,- 068 & 213 &,- 049 \\
\hline Importancia de formarse su propia opinión personal &,- 095 & 076 &,- 006 & ,079 & 600 &,- 089 &, 173 &,- 095 & ,094 \\
\hline Importancia votar en las elecciones &, 213 & ,096 &,- 019 & 083 & ,594 & ,024 & ,056 & 354 & 044 \\
\hline Importancia pensar en los demás & .139 &, 034 &, 044 &,- 060 &, 547 &,- 111 & 153 &,- 127 & .060 \\
\hline Ir a una manifestación no sirve para nada &,- 186 & 043 &,- 022 &,- 057 &,- 008 &, 575 &,- 265 &,- 269 &,- 017 \\
\hline Esté quién esté al poder sólo busca sus intereses personales &,- 256 &,- 223 &,- 028 &,- 180 &,- 111 & .570 & 163 & 085 & 109 \\
\hline Ir a una manifestación requiere mucho tiempo y esfuerzo &,- 005 &, 044 &, 032 &, 034 & ,096 &, 554 &,- 172 &,- 130 &,- 155 \\
\hline Los políticos no se preocupan de lo que piensa la gente normal &,- 302 &,- 170 & 007 &,- 094 &,- 137 & ,503 & 133 & 139 & .119 \\
\hline La política es complicada de entender & ,068 &,- 451 &,- 015 &,- 090 &,- 060 & ,464 &,- 107 & 009 & 080 \\
\hline Activismo en los últimos 12 meses &, 023 & 190 &, 012 & ,332 & .064 &,- 084 &, 587 & 158 & .116 \\
\hline Alguien le ha comentado que había una manifestación y le convenció para ir &,- 001 &, 164 &,- 007 & .129 &,- 001 &,- 007 & .577 & .076 & 015 \\
\hline Actitud acerca implicación ciudadana en la toma de decisiones políticas &, 033 &,- 068 & ,009 &,- 031 &, 054 &,- 008 & 408 &,- 014 &,- 059 \\
\hline Ha recibido mensaje de móvil para asistir a manifestación &,- 012 & ,072 & ,048 & 272 &,- 040 &,- 090 & ,395 &,- 042 & .009 \\
\hline Confianza en la gente & , 198 & 079 & ,068 &,- 058 &, 058 &,- 108 & 334 &, 075 &,- 260 \\
\hline Voto en las últimas elecciones & ,007 & 159 & 023 &, 000 & 125 &,- 062 & 067 & .567 &,- 027 \\
\hline Le resulta difícil decidir para quién ir a votar &,- 022 &,- 091 &, 042 &,- 160 & ,009 & 249 &, 104 &,- 559 & 098 \\
\hline Votar es fácil y no requiere ningún esfuerzo & .010 &,- 013 &,- 015 &, 082 & 027 & 078 & ,078 &, 532 & ,047 \\
\hline Mi voto es inútil porque no cambia el resultado de las elecciones &,- 082 &,- 093 &,- 025 &,- 067 &,- 134 & 437 &,- 144 &,- 464 & 006 \\
\hline Disposición voto a través de Internet & 112 & 044 &, 002 &,- 008 &,- 031 & 004 & 206 &,- 055 &,- 772 \\
\hline Percepción de falta de seguridad de Internet &,- 009 & 092 & ,037 &,- 005 & ,091 &,- 015 & 124 &,- 056 & ,718 \\
\hline
\end{tabular}

Fuente: Elaboración propia a partir de los datos del CIS (estudio 2736). 
El factor 6 se ha interpretado como Percepción de falta de eficacia política. Éste se compone de variables que anteriormente se habian incluido en las dimensiones del Cinismo (como, por ejemplo, "Ir a una manifestación no sirve para nada" o "Esté quién esté al poder sólo busca sus intereses personales") y de la Eficacia de la información política (por ejemplo: "La política es complicada de entender").

Las variables inherentes el activismo, los estímulos movilizadores difundidos a través de sms o de otra persona, la actitud positiva hacia la implicación ciudadana y la confianza en la gente contribuyen en el factor 7 , interpretado como Nuevas formas de participación política.

El factor 8 está compuesto por la participación en las últimas elecciones, por la percepción de utilidad del voto y por la percepción de facilidad de la puesta en marcha de la actividad de voto. Este factor se ha interpretado como Actitud proactiva hacia el voto.

Finalmente, el factor 9 se compone por la percepción de falta de seguridad de Internet y la no disposición para el voto a través de esta herramienta. Se ha interpretado este factor como Actitud negativa hacia el uso de Internet para la participación política.

Estas dimensiones han sido empleadas como variables en el siguiente análisis de regresión logística. De hecho, estas nueve variables son estandarizadas, lo cual garantiza la posibilidad de comparar directamente los índices de regresión asociados a cada una de ellas.

\subsection{Regresión logística}

Este análisis ha sido implementado para averiguar las influencias, sobre el uso de blogs políticos, debidas a variables políticas, de consumo de información, de estímulos movilizadores, de las variables socio demográficas y de los distintos usos de Internet.

El modelo de regresión tiene como variables independientes los factores extraídos por el análisis anterior, el sexo, la edad, el nivel de estudios, el estatus socioeconómico y los usos de internet (ver arriba). La variable dependiente coincide con la pregunta P28(5) del estudio 2736 del CIS sobre uso de blogs políticos.
En primer lugar, cabe destacar que los test ómnibus de los coeficientes del modelo son todos significativos. La $\mathrm{R}^{2}$ de Cox y Snell es de 0,262, mientras que la $R^{2}$ de Nagelkerke es de 0,406.

El modelo permite predecir correctamente el $83,1 \%$ de los casos (con un punto de corte de 0,5 ). Más en concreto, permite predecir el 93,9\% de los casos negativos y el 43,5\% de los positivos. Se detecta cierta desproporción entre la predicción de casos negativos y la de casos positivos. Es posible que esto se deba al hecho de que la pregunta que mide la variable dependiente hace referencia a más de una conducta. Consecuentemente, es posible que no se hayan incluido factores o variables explicativas inherentes a los foros 0 las páginas web y que hubiesen permitido explicar más casos positivos.

A continuación se presenta la tabla 6 con las variables introducidas en la ecuación y los coeficientes a ellas correspondientes.

En primer lugar, es posible destacar que, entre las variables sociodemograficas, sólo la edad es significativa ( $p$-valor menor de 0,05) incidiendo negativamente sobre la posibilidad de escribir comentarios sobre un blog político.

En relación a los usos de Internet, las únicas variables significativas son "Intervenir en chats 0 en foros de discusión", "Mantener su propio blog o página web" y "Navegar por Internet sin un objetivo concreto": Siendo las tres variables dicotómicas es posible confrontar directamente los coeficientes $B$. Éstos nos informan de que las influencias son todas positivas y de que la influencia más importante llega del hecho de escribir en chats 0 foros. La segunda variable más influyente, dentro de los usos de Internet, es navegar por Internet sin un objetivo concreto.

Respecto a los factores, sólo hay tres que resultan ser significativos en este modelo. Los tres tienen un efecto positivo. Siendo variables estandarizadas, es posible comparar directamente los coeficientes B. Así, el factor más influyente resulta ser la participación política institucional. El segundo factor más influyente es representado por las nuevas formas de participación. La actitud proactiva hacia la política es el tercer factor en orden de influencia. 


\begin{tabular}{|c|c|c|c|c|c|c|c|}
\hline & & & & & & \\
\hline & & B & D.S. & Wald & gdl & Sig. & $\operatorname{Exp}(B)$ \\
\hline \multirow{13}{*}{ 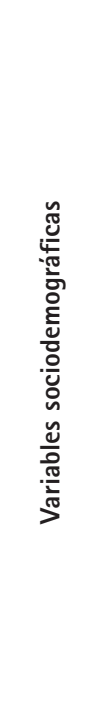 } & Sexo(1) & $-8,341 \mathrm{E}-02$ & 174 & 231 & 1 & ,631 & 920 \\
\hline & Edad* $^{*}$ & $-2,559 \mathrm{E}-02$ & 010 & 6,977 & 1 & ,008 & 975 \\
\hline & NIVEL DE ESTUDIOS & & & 5,989 & 5 & ,307 & \\
\hline & NIVEL DE ESTUDIOS(1) & 18,969 & $16.746,701$ & 000 & 1 & ,999 & $1,731 \mathrm{E}+08$ \\
\hline & NIVEL DE ESTUDIOS(2) & 19,481 & $16.746,701$ & ,000 & 1 & ,999 & $2,888 \mathrm{E}+08$ \\
\hline & NIVEL DE ESTUDIOS(3) & 19,314 & $16.746,701$ & ,000 & 1 & ,999 & $2,442 E+08$ \\
\hline & NIVEL DE ESTUDIOS(4) & 19,509 & $16.746,701$ & 000 & 1 & ,999 & $2,968 \mathrm{E}+08$ \\
\hline & NIVEL DE ESTUDIOS(5) & 19,129 & $16.746,701$ & ,000 & 1 & ,999 & $2,031 E+08$ \\
\hline & Condición socioeconómica & & & 1,110 & 4 & 893 & \\
\hline & Condición socioeconómica(1) & 137 & 219 & ,393 & 1 &, 531 & 1,147 \\
\hline & Condición socioeconómica(2) & 039 &, 318 &, 015 & 1 & ,901 & 1,040 \\
\hline & Condición socioeconómica(3) &, 108 & 257 & 177 & 1 &, 674 & 1,114 \\
\hline & Condición socioeconómica(4) & $-1,653 \mathrm{E}-01$ &, 344 & 231 & 1 & 631 & ,848 \\
\hline \multirow{9}{*}{ 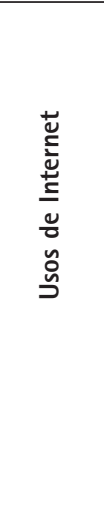 } & Buscar Información (trabajo, estudios, salud, etc.) (1) &, 745 &, 643 & 1,341 & 1 & 247 & 2,106 \\
\hline & Comprar algún producto o servicio (1) & 258 &, 172 & 2,260 & 1 & 133 & 1,294 \\
\hline & Realizar gestiones bancarias (1) & 035 & 175 &, 041 & 1 & 840 & 1,036 \\
\hline & Recibir o enviar mensajes de correo electrónico (1) & 631 &, 424 & 2,211 & 1 &, 137 & 1,879 \\
\hline & Intervenir en chats o foros de discusión (1)* & 1,188 &, 166 & 51,333 & 1 & $7,795 \mathrm{E}-13$ & 3,280 \\
\hline & Llamar a través de Internet (1) &, 070 &, 194 &, 131 & 1 &, 718 & 1,073 \\
\hline & Descargar archivos (1) & .423 & 279 & 2,293 & 1 &, 130 & 1,527 \\
\hline & Mantener su proprio blog o página web* (1) &, 550 & 172 & 10,286 & 1 & 001 & 1,734 \\
\hline & Navegar por internet sin un objetivo concreto* (1) &, 741 & 210 & 12,457 & 1 &, 000 & 2,097 \\
\hline \multirow{10}{*}{ 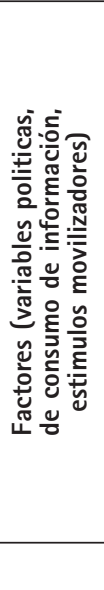 } & Confianza en las instituciones & 022 & 079 & 079 & 1 &, 778 & 1,023 \\
\hline & Actitud proactiva hacia la política* & 471 & 086 & 29,873 & 1 & $4,614 \mathrm{E}-08$ & 1,601 \\
\hline & Lectura de prensa &, 024 & 084 & 079 & 1 &, 779 & 1,024 \\
\hline & Participación política institucional* & 648 & 078 & 68,891 & 1 & $1,040 \mathrm{E}-16$ & 1,912 \\
\hline & Actitudes ciudadanas &, 004 & ,080 &, 003 & 1 & 957 & 1,004 \\
\hline & Percepción de falta de eficacia política & $-9,981 E-03$ & 079 &, 016 & 1 & 900 & ,990 \\
\hline & Nuevas formas de participación política* &, 525 & ,083 & 39,642 & 1 & $3,051 \mathrm{E}-10$ & 1,690 \\
\hline & Actitud proactiva hacia el voto & $-9,247 \mathrm{E}-02$ & 084 & 1,211 & 1 & 271 & ,912 \\
\hline & $\begin{array}{l}\text { Actitud negativa hacia el uso de Internet para la } \\
\text { participación política }\end{array}$ & $5,949 \mathrm{E}-02$ & 081 &, 538 & 1 & .463 &, 942 \\
\hline & Constante & $-2,305 E+01$ & $16.746,701$ &, 000 & 1 & ,999 & $9,758 \mathrm{E}-11$ \\
\hline
\end{tabular}

Fuente: Elaboración propia a partir de los datos de la encuesta 2736 del CIS. 


\section{INTERPRETACIÓN DE LOS RESULTADOS}

El análisis ha destacado que, entre las variables sociodemográficas escogidas, sólo la edad tiene una relación significativa con la variable dependiente. Este resultado puede tener una explicación en el hecho de que se han excluido del análisis los no internautas. Consecuentemente, se han podido excluir efectos debidos a la brecha digital de primer nivel. De hecho, según los estudios empíricos sobre este fenómeno (DiMaggio, 2001; Attewell, 2000), sabemos que, sobre el acceso a Internet, influyen variables sociodemograficas como el sexo, el nivel de estudios, el nivel de renta o la raza. Estos factores parecen ser, excluida la edad, no relevantes en la explicación de un comportamiento político online como escribir comentarios en blogs de naturaleza política. Aún así, los internautas de mayor edad parecen tener menos probabilidades de utilizar políticamente esta herramienta. Consecuentemente, es razonable pensar que esta actividad política online se distribuye de manera desigual entre la población española. Esto nos lleva a razonar sobre este fenómeno desde una perspectiva de brecha digital de segundo nivel.

Como se ha mencionado anteriormente, los usos de Internet han sido introducidos en el modelo de regresión para poder averiguar si hay alguna actividad concreta que se realice a través de Internet que pueda facilitar el hecho de escribir en un blog de contenido político. Esto hubiera permitido hacer alguna reflexión acerca de posibles efectos negativos debidos a la brecha digital de segundo nivel.

Los resultados ponen de manifiesto, en primer lugar, el hecho de que conductas como intervenir en chats o foros y mantener un blog o una página web influyen en la conducta estudiada. Es razonable pensar que el hecho de escribir comentarios en foros, aunque de contenido no político, y mantener un blog, hagan del sujeto un usuario habitual de la herramienta, facilitando así su uso también en el ámbito político.

Sin embargo, el segundo tipo de uso de Internet más influyente del modelo de regresión es el navegar sin un objetivo concreto. Es un uso considerado de diversión (Shah et al., 2001). Aun así tiene influencia sobre la probabilidad de escribir comentarios en un blog político. Esto podría apoyar a la hipótesis de que Internet provea información política no solicitada (Horrigan et al., 2004). Si esto fuese cierto, sería una evidencia a favor del potencial innovador democrático de Internet.

De todos los factores introducidos en el modelo de regresión logística, sólo resultan ser significativas la participación política institucional, las nuevas formas de participación política y la actitud proactiva hacia la política.

Antes de analizar la incidencia de estos factores es oportuno reflexionar sobre la pregunta que mide la variable dependiente. Ésta ha sido formulada como "¿En alguna ocasión ha escrito comentarios en algún foro, blog o página web, sobre temas de actualidad, sociales o políticos?". Esta formulación, a pesar de las limitaciones destacadas anteriormente, implica el uso comunicativo del blog y no el informativo o el contra-informativo.

Es razonable pensar que esto pueda ser la razón de la no significatividad de factores como el uso de prensa, la confianza en las instituciones o la percepción de falta de eficacia política, más conectados al segundo tipo de uso.

Con respecto a la influencia de los factores que han resultado ser significativos, el análisis ha destacado la mayor influencia de la participación política institucional. Aunque esto pueda parecer aportar elementos a favor de la teoría de la normalización, destacando una continuidad de los tipos clásicos de participación política en el ámbito online, es importante precisar dos asuntos. En primer lugar, también tienen influencia las nuevas formas de participación política. Esto podria ser una señal de que esta herramienta da realmente oportunidad de desarrollo en la red tanto a las prácticas tradicionales como a las nuevas y emergentes, manteniendo así su potencial democratizador.

En segundo lugar, la investigación sobre los blogs de los representantes políticos parece evidenciar un cambio en la gestión tradicional de las funciones de representación política. Si bien la mayoría de los partidos políticos tienen, hoy en dia, una página web (véase, por ejemplo, Gibson y Ward, 2002), la decisión de utilizar un blog implica la intención de mantener otro tipo de relación y de comunicación entre representante y representados. Por ejemplo, según los resultados obtenidos por Jackson (2008), los parlamentarios no utilizan un blog para apoyar las funciones desempeñadas fuera de línea, tales como su rol de circunscripción. Más bien, un blog parece tener un papel 
muy limitado y específico de promoción del debate dentro de una e-circunscripción separada. Wright, analizando la experiencia británica de Read My Day (2008), concluye que, si es cierto que la comunicación política es cada vez más negativa, orientada al ataque, dominada por los dirigentes políticos y carente de sustancia, los blogs hacen, en cierto modo, que esto se corrija, representando así un modo no convencional de hacer política. Coleman (2004) ha conseguido demostrar que las consultas online proveen espacio para una deliberación pública inclusiva, además de crear y conectar redes de interés o prácticas.

\section{Conclusiones}

En este trabajo se han estudiado, según una perspectiva exploratoria, algunos de los determinantes del uso de blogs de contenido político por parte de los internautas.

Utilizando los datos del estudio del CIS "Internet y participación política", se ha realizado un análisis factorial que incluyese las variables políticas y de consumo de información estudiadas en la literatura sobre blogs.

Las dimensiones extraídas han sido introducidas en un modelo de regresión logística como variables independientes junto con los usos de internet y las variables sociodemográficas sexo, edad, estatus socioeconómico y nivel de estudios. La variable dependiente ha coincidido con el uso de blogs políticos. La muestra elegida han sido los internautas españoles.

Los resultados indican que las variables socio-demográficas, menos la edad, no resultan significativas. A nivel de variables políticas, se destaca la influencia de la participación institucional y de las nuevas formas de participación. Esto implicaria el hecho de que estas herramientas puedan propiciar la difusión de prácticas políticas que en el "mundo real" tienen menor relevancia política.

Además, se ha destacado que el hecho de utilizar Internet sin un objetivo concreto presenta una particular influencia sobre el hecho de escribir comentarios en blogs políticos. Esto podría implicar una extensión de esta herramienta también a los usuarios políticamente menos interesados, debido al hecho de que la información política online llega también de forma indirecta al usuario.

Es importante que las indicaciones que resultan de este trabajo se tomen como un primer acercamiento a la investigación sobre los blogs políticos.

En primer lugar, porque la variable dependiente sólo toma en consideración la conducta comunicativa realizable a través de estas plataformas, pero no las informativas y contra-informativas.

En segundo lugar, porque en el análisis no se han incluido variables inherentes a la capacidad del uso de la herramienta Internet y de los recursos informáticos, así como no se ha incluido el capital social. Es razonable pensar que estas dos dimensiones puedan contribuir en la explicación del uso de blogs políticos.

Además, la formulación de la pregunta que mide la variable dependiente se centra también en otras plataformas como foros o páginas web. Consecuentemente no se pueden excluir otros factores explicativos no tomados en cuenta en este trabajo.

\section{NOTAS}

1 Muestreo polietápico y estratificado por conglomerados. Afijación de tipo no proporcional. La utilización de la muestra en su conjunto ha implicado

Recibido: 1 de julio de 2011

Aceptado: 15 de enero de 2012 el uso de ponderación. Para un nivel de confianza del 95,5\% (dos sigmas), y $P=0$, el error real es de $\pm 1,64 \%$ para el conjunto de la muestra y en el supuesto de muestreo aleatorio simple (Fuente: Ficha Técnica del CIS, estudio 2736).

2 El cuestionario completo es consultable en la página web del CIS: www. cis.es. 


\section{BIBLIOGRAFÍA}

Anduiza, E.; Cantijoch, M.; Colombo, C.; Gallego, A. y Salcedo, J. (2010): "Los usos políticos de Internet en España", Revista Española de Investigaciones Sociológicas, 129(-1), 133-146.

Attewell, P. (2001): "Comment: The first and second digital divides", Sociology of education, 74(3), 252-259.

Bar-llan, J. (2005): "Information hub blogs", Journal of Information Science, 31(4), 297.

Best, S. y Krueger, B. (2005): "Analyzing the representativeness of Internet political participation", Political Behavior, 27(2), 183-216.

Bimber, B. y Davis, R. (2003): Campaigning online: The Internet in US elections, Oxford University Press, USA.

Bimber, B. (2001): "Information and political engagement in America: The search for effects of information technology at the individual level", Political Research Quarterly, 54(1), 53.

Bonfadelli, H. (2002): "The Internet and knowledge gaps", European Journal of communication, 17(1), 65.

Bruns, A. (2008): "Life beyond the public sphere: Towards a networked model for political deliberation", Information Polity, 13(1), 71-85.

Cliff, N. (1988): "The eigenvalues-greaterthan-one rule and the reliability of components", Psychological Bulletin, 103, 276-279.

Coleman, S. y Wright, S. (2008): "Political blogs and representative democracy", Information Polity, 13(1), 1-6.

Coleman, S. (2004): "Connecting Parliament to the Public via the Internet", Information, Communication \& Society, 7(1), 1-22.

Coleman, S. (2005): "Blogs and the new politics of listening", Political Quarterly, 76(2), 272-280.
Colombo, C. (2007): Innovación democrática y TIC ¿Hacia una democracia participativa?, Universitat Oberta de Catalunya.

Cornfield, M. (2000): "A user's guide to the digital divide", Campaigns and Elections, 21 (3), 47.

Dahlberg, L. (2001): "Democracy via cyberspace", new media \& society, 3(2), 157.

De Zuniga, H. G.; Puig i Abril, E. y Rojas, H. (2009): "Weblogs, traditional sources online and political participation: an assessment of how the internet is changing the political environment", New Media \& Society, 11(4), 553.

De Zuñiga, H.; Veenstra, A.; Vraga, E.; Wang, M.; DeShano, C.; Perlmutter, D. y Shah, D. (2007): "Online and Offline Activism: Communication Mediation and Political Messaging Among Blog Readers", Annual convention of the Association for Education in Journalism and Mass Communication, Washington, DC, 9-12.

DiMaggio, P.; Hargittai, E.; Neuman, W. y Robinson, J. (2001): "Social implications of the Internet", Annual review of sociology, 27, 307-336.

Drezner, D. y Farrell, H. (2004): "Web of influence", Foreign Policy, 32-41.

Dunham, R. (1999): "Across America, a troubling 'digital divide'", Business Week, 3640(2).

Eveland, W.; Hayes, A.; Shah, D. y Kwak, N. (2005): "Understanding the relationship between communication and political knowledge: A model comparison approach using panel data", Political Communication, 22(4), 423-446.

Ferguson, R. y Griffiths, B. (2006): "Thin democracy? Parliamentarians, citizens and the influence of blogging on political engagement", Parliamentary Affairs, 59(2), 366.

Gibson, R. y Ward, S. (2002): "Virtual campaigning: Australian parties and the impact of the Internet", Australian Journal of Political Science, 37(1), 99-129.

Gibson, R.; Lusoli, W. y Ward, S. (2005): "Online Participation in the UK: Testing a 'Contextualised' Model of Internet Effects", The British Journal of Politics \& International Relations, 7(4), 561-583.

Gillmor, D. (2004): We the Media, O'Reilly Media, Inc.

Hacker, K. y Van Dijk, J. (2001): Digital democracy: Issues of theory and practice, Sage Publications, Inc. Thousand Oaks, CA, USA.

Hargittai, E. (2002): "Differences in people's online skills", First Monday, 7(4-1).

Harman, H. H. (1976): Modern factor analysis, Chicago: University of Chicago Press.

Hershey, M. y Hill, D. (1975): "Watergate and preadults' attitudes toward the President", American Journal of Political Science, 703-726.

Horrigan, J.; Garrett, K. y Resnick, P. (2004): The Internet and democratic debate, Pew Internet \& American Life Project.

Hsu, C. y Lin, J. (2008): "Acceptance of blog usage: The roles of technology acceptance, social influence and knowledge sharing motivation", Information \& Management, 45(1), 65-74.

Hwang, H.; Schmierbach, M.; Paek, H.; de Zúñiga, H. y Shah, D. (2006): "Media dissociation, Internet use, and antiwar political participation: A case study of political dissent and action against the war in Iraq", Mass Communication and Society, 9(4), 461483.

Jackson, N. (2008): "'Scattergun' or 'rifle' approach to communication: MPs in the blogosphere", Information Polity, 13(1), 57-69.

Johnson, T. y Kaye, B. (2004): "Wag the blog: How reliance on traditional media 
and the Internet influence credibility perceptions of weblogs among blog users", Journalism and Mass Communication Quarterly, 81, 622-642.

Johnson, T.; Kaye, B.; Bichard, S. y Wong, W. (2007): "Every blog has its day: Politically-interested Internet users' perceptions of blog credibility", Journal of Computer-Mediated Communication, 13(1), 100-122.

Kahn, R. y Kellner, D. (2004): "New media and internet activism: from the 'Battle of Seattle' to blogging", New Media \& Society, 6(1), 87.

Kaid, L.; McKinney, M. y Tedesco, J. (2007): "Political information efficacy and young voters", American Behavioral Scientist, 50(9), 1093-1111.

Krueger, B. (2002): "Assessing the potential of Internet political participation in the United States", American Politics Research, 30(5), 476.

Lawley, D. N. y Maxwell, A. E. (1971): Factor Analysis as a Statistical Method, 2nd edition. London: Butterworth.

Lewis-Beck, M. (1994): Factor analysis and related techniques, Sage London.

Livingstone, S. y Helsper, E. (2007): "Gradations in digital inclusion: children, young people and the digital divide", New Media \& Society, 9(4), 671.

Margolis, M. y Resnick, D. (2000): Politics as usual: the cyberspace "revolution", Sage.

Miller, A. (1974): "Political issues and trust in government: 1964-1970", The American Political Science Review, 68(3), 951-972.
Mossberger, K.; Tolbert, C. y Stansbury, M. (2003): Virtual inequality: Beyond the digital divide, Georgetown Univ Pr.

Mutz, D.; Martin, P. (2001): "Facilitating communication across lines of political difference: The role of mass media", American Political Science Review, 95(1), 97-114.

Nardi, B.; Schiano, D. y Gumbrecht, M. (2004): "Blogging as social activity, or, would you let 900 million people read your diary?" "Proceedings of the 2004 ACM conference on Computer supported cooperative work", ACM, 222-231.

Norris, P. (2001): Digital divide: Civic engagement, information poverty, and the Internet worldwide, Cambridge Univ Pr.

Norris, P. (2003): "Preaching to the Converted?", Party Politics, 9(1), 21.

Robinson, J.; DiMaggio, P. y Hargittai, E. (2003): "New social survey perspectives on the digital divide", It \& Society, 1(5), 1-22.

Robles, J. (2008): Ciudadanía digital: una introducción a un nuevo concepto de ciudadano, Editorial UOC.

Schuefele, D. y Nisbet, M. (2002): "Being a Citizen Online: New Opportunities and Dead Ends", The Harvard Journal of Press/Politics, 7(3), 55-75.

Shah, D.; Kwak, N. y Holbert, R. (2001): "'Connecting' and 'disconnecting' with civic life: Patterns of Internet use and the production of social capital", Political Communication, 18(2), 141-162.

Singer, J. (2005): "The political j-blogger", Journalism, 6(2), 173.
Subirats, J. (2002): "Los dilemas de una relación inevitable. Innovación democrática y tecnologías de la información y de la comunicación", contenido en Cairo Carou, H. (Comp.), Democracia Digital. Límites y oportunidades, pp. 89-111, Madrid, Trotta.

Sweetser, K. y Lee Kaid, L. (2008): "Stealth soapboxes: political information efficacy, cynicism and uses of celebrity weblogs among readers", New Media \& Society, 10(1), 67.

Wallsten, K. (2008): "Political blogs: Transmission belts, soapboxes, mobilizers, or conversation starters?", Journal of Information Technology\& Politics, 4(3), 19-40.

Warschauer, M. (2003): "Demystifying the digital divide", Scientific American, 289(2), 34-39.

Wasserman, I. y Richmond-Abbott, M. (2005): "Gender and the Internet: Causes of Variation in Access, Level, and Scope of Use", Social Science Quarterly, 86(1), 252-270.

Woodly, D. (2008): "New competencies in democratic communication? Blogs, agenda setting and political participation", Public Choice, 134(1), 109-123.

Wright, S. (2008): "Read My Day? Communication, campaigning and counciIlors' blogs", Information Polity, 13(1), 41-55.

Xenos, M. (2008): "New mediated deliberation: Blog and press coverage of the Alito nomination", Journal of Computer-Mediated Communication, 13(2), 485-503. 